(c) Auteures. Cette œuvre, disponible à http://dx.doi.org/10.18162/fp.2020.698, est distribuée sous licence Creative Commons Attribution 4.0 International http://creativecommons.org/licences/by/4.0/deed.fr

\section{Influences et répercussions de la pandémie sur les apprentissages et le parcours universitaire des étudiant-e.s aux cycles supérieurs en sciences de l'éducation : retour d'expérience de trois doctorantes}

Influences and repercussions of the pandemic on the learning and

Marjorie Cuerrier

Anne-Sophie Aubin

Delphine Tremblay-Gagnon

Université de Montréal (Canada) university pathways of graduate students in education sciences:

experiential accounts of three doctoral students

doi: 10.18162/fp.2020.698

\section{ésumé}

Lors de cette pandémie mondiale sans précédent, le domaine de l'éducation a été particulièrement touché alors que les établissements d'enseignement n'ont eu d'autre choix que de fermer leurs portes en présentiel à plus de 1,6 milliard d'apprenant.e.s et 60 millions d'enseignant-e.s à travers le monde. Les établissements universitaires n'y faisant pas

exception, les influences et les répercussions sont notables chez les étudiant.e.s universitaires,

entre autres chez les étudiant.e.s aux cycles supérieurs en sciences de l'éducation. Dans ce retour d'expérience de trois doctorantes, trois axes phares ont été établis : la santé psychologique, les conditions de travail et les tâches.

Mots-clés

COVID-19, retour d'expérience, santé psychologique, conditions de travail, tâches

Abstract

Since the start of the pandemic, the education field has been particularly hard hit. Schools had no choice but to close their doors to over 1.6 billion students and 60 million teachers worldwide from

March to May 2020. Nor did universities escape the influences and repercussions of these closures, with notable impacts on graduate students in the education sciences. Three doctoral students share their stories of the experience, revealing three predominantly affected areas: psychological health, working conditions, and tasks.

Keywords

COVID-19; experiential account, psychological health, working conditions, tasks

\section{Introduction}

Depuis mars 2020, les normes et les conventions qui régissent les sphères personnelles, professionnelles et scolaires de tout un chacun sont bouleversées par cette pandémie mondiale sans précédent. En réponse à celle-ci, des actions et des mesures sont mises en place, entraînant ainsi des transitions drastiques : des rencontres physiques aux rencontres virtuelles, du travail au bureau au télétravail, de l'enseignement en présentiel à l'enseignement à distance, les différentes facettes de ces sphères sont repensées pour freiner la propagation de la COVID-19 et assurer la sécurité de tous. Cela dit, sans s'y restreindre, l'éducation est l'un des domaines les plus sévèrement touchés par la crise alors que les établissements d'enseignement primaire, secondaire, collégial et universitaire ont fermé temporairement leurs portes en présentiel à plus de 1,6 milliard d'apprenant·ess et 60 millions d'enseignant·e's à travers le monde (UNESCO, 2020).

En plus d'être tangibles, les influences et les répercussions de la pandémie à tous ordres d'enseignement sont variées considérant la réalité et les défis spécifiques avec lesquels les établissements doivent composer. De fait, puisque le curriculum, les objectifs de formation et les compétences à développer diffèrent, un regard nuancé et précis est de mise afin de brosser un portrait du domaine à tous les ordres d'enseignement. Étant doctorantes en sciences de l'éducation au moment de la pandémie et titulaires de postes décisionnels à l'Association des étudiant'e's aux cycles supérieurs en éducation (ACSE) de l'Université de Montréal, nous sommes des témoins privilégiés de ces influences et de ces répercussions sur les apprentissages et sur le parcours académique des étudiant·e's aux cycles supérieurs en éducation. Actuellement, au Québec, les cycles supérieurs représentent près de 76000 étudiant ${ }^{\cdot} \cdot s$, soit environ 59000 étudiant $\cdot \cdot \cdot s$ au $2^{\mathrm{e}}$ cycle et 17000 étudiant·e’s au 3e cycle (Gouvernement du Québec, 2019). 
Ce nombre représente $25 \%$ de la clientèle universitaire, un pourcentage qui s'avère non négligeable et de celui-ci, un nombre important d'étudiant·e's est issu des sciences de l'éducation. Ainsi, dans le cadre de cet article, les influences et les répercussions de la pandémie sur ces étudiant·e•s universitaires sont étayées afin de rendre compte de certains avantages et défis y étant associés. Trois axes phares sont proposés : la santé psychologique, les conditions de travail et les tâches.

\section{La santé psychologique : stress, incertitudes et vie aux cycles supérieurs}

Une pandémie mondiale peut représenter un stresseur important pour tous considérant les incertitudes liées aux sphères personnelles et professionnelles. Cela étant dit, les étudiant·e's aux cycles supérieurs en sciences de l'éducation n'échappent pas à ces incertitudes et doivent aussi conjuguer avec celles liées à la sphère académique (p. ex. : annulation de cours, transfert de ceux-ci en ligne, changement du barème de notation, etc.). Précisons néanmoins que le niveau de stress vécu et ressenti ainsi que les façons de gérer ce stress diffèrent d'une personne à l'autre. Dans tous les cas, c'est dans un climat anormal que le trimestre d'hiver 2020 s'est achevé et que les étudiant·e·s ont entamé leur trimestre d'été. Alors que l'été est, pour plusieurs, une pause estivale leur permettant de se reposer, d'écrire et d'avancer leurs travaux de maîtrise ou de doctorat, en 2020, la situation est tout autre. En ce sens, dans ce climat de bouleversements et d'incertitudes liés aux différentes sphères de vie, ils sont amenés à consulter de façon fréquente l'actualité afin de connaitre l'évolution de la situation pandémique et des mesures exceptionnelles à adopter. À titre d'exemple, durant cette période pandémique, nous estimons avoir consulté nous-mêmes l'actualité une douzaine de fois par jour via des médias variés : télévision, journaux, réseaux sociaux, courriel, etc., une moyenne nettement supérieure à l'habitude.

Toujours dans cette optique, selon plusieurs chercheurs, dont Holman, Garfin et Silver (2014) ainsi que Johnston et Davey (1997), s'informer quotidiennement sur l'actualité pourrait avoir des effets négatifs sur un individu. De surcroît, en contexte de pandémie, il va sans dire que l'actualité quotidienne est affligeante à la lumière des statistiques funestes présentées. Ces chercheurs avancent justement que ces effets négatifs peuvent s'apparenter aux symptômes de l'anxiété, tels que la peur et le sentiment d'impuissance, mais aussi des difficultés à se concentrer ou à trouver le sommeil. Dans certains cas, cela pourrait également exacerber les préoccupations personnelles n'ayant, au départ, aucun lien avec la situation observée (Johnston et Davey, 1997). Dans le même ordre d'idées, une personne est susceptible de devenir très vigilante face à ce stresseur important (ici la pandémie), car le cerveau se concentre sur la «menace détectée » (Lupien, 2020) et tous les petits pépins quotidiens peuvent alors être poussés à leur paroxysme. À titre d'exemple, pour un étudiant·e aux cycles supérieurs en sciences de l'éducation, cela peut consister en plusieurs choses : ne pas réussir à avancer son chapitre de discussion, avoir de la difficulté à écrire la dernière ligne de son devis de recherche pourtant si bien entamé, etc.

De plus, considérant l'aspect exceptionnel de la situation, peu de personnes peuvent affirmer avoir déjà vécu des situations semblables et cela amène un lot d'incertitudes et de nouveautés pour la plupart des individus. Autrement dit, nous sommes d'avis que le contexte pandémique vécu et diffusé dans l'actualité est susceptible, tant par son aspect anxiogène qu'inconnu, d'influencer la santé psychologique des étudiant'e's aux cycles supérieurs en sciences de l'éducation. Les effets négatifs y étant associés pourraient se refléter entre autres dans un déséquilibre entre la vie personnelle et professionnelle ainsi que dans la perte de points de repère importants, comme nous l'abordons dans les prochaines sections.

2 - Formation et profession 28(4 hors-série), 2020 


\section{La situation de vie et le rapport à la solitude en contexte de confinement}

Afin de limiter la propagation de la COVID-19, un confinement volontaire a été recommandé pendant plusieurs semaines par les différents paliers gouvernementaux au printemps 2020. Bien que profitable pour la santé publique, cette recommandation est susceptible d'influencer les apprentissages et le parcours universitaire des étudiant·e's aux cycles supérieurs en sciences de l'éducation à court et long termes. De fait, dans ses billets quotidiens publiés lors de la pandémie, la chercheuse Sonia Lupien aborde plusieurs thématiques relatives au stress vécu dans ce contexte, entre autres quant à la situation de vie et au rapport à la solitude ${ }^{1}$. De manière générale, Lupien souligne que vivre seul·e lors de la pandémie est susceptible d'augmenter le stress ressenti, ce qui est le cas, supposons-le, pour plusieurs étudiant·e·s. De surcroît, en plus de se retrouver seul·e·s à leur domicile et sujet·te's à un stress augmenté, bon nombre d'étudiant·e's sont coupé·e's de leur cercle de soutien social habituel (amis, collègues de travail, direction de recherche, équipe de recherche, membres du personnel académique, etc.). Pour certain'e's, cette situation d'isolement est très difficile et pénible à vivre, comme en témoigne la démultiplication des demandes d'aide, de soutien et d'accompagnement formulées à l'Association des étudiant $\cdot$ 's aux cycles supérieurs en éducation (ACSE) lors de cette période. Dans cette suite d'idées, nous n'excluons pas la possibilité que le stress vécu puisse possiblement ralentir, voire freiner, l'avancement académique de certains étudiant·e·s.

Cependant, il ne faut pas croire que le fait de vivre le confinement avec d'autres personnes ne comporte que des avantages. En effet, Lupien, toujours dans ses billets sur la COVID-19, souligne que plusieurs études ont révélé que le stress est susceptible d'augmenter en situation de surpeuplement (Lin, Sun, Choi, Magee, Stets et During, 2015) et que le confinement volontaire vécu lors de la pandémie peut s'apparenter à ce type de surpeuplement si plusieurs individus vivent sous un même toit (p. ex., avec des conjoint $\cdot$ •`s, enfants, parents, colocataires, membres de la famille, etc.). De plus, vivre dans une situation de surpeuplement peut causer d'autres inconvénients, entre autres limiter la concentration et l'efficacité à travailler considérant les possibles dérangements et les arrêts fréquents. Le sentiment de performance peut alors être diminué, affectant la perception de notre valeur en tant qu'étudiant·echercheur·e. En bref, de la solitude au surpeuplement, toute situation de confinement a le potentiel d'augmenter considérablement le stress ressenti par les étudiant·e·s aux cycles supérieurs en sciences de l'éducation et d'affecter la santé psychologique de ceux-ci.

\section{La gestion du temps, une habileté à redéfinir}

La gestion du temps est également un élément d'intérêt lié à la santé psychologique et dont les influences et les répercussions de la pandémie se font ressentir chez ces étudiant·e·s, selon notre analyse. Alors que la plupart des activités professionnelles et scientifiques ont été annulées pour limiter la propagation de la COVID-19, plusieurs étudiants en profitent pour avancer leurs travaux premiers : leur mémoire ou leur thèse. Dispensés de plusieurs autres tâches connexes, certains accueillent de façon favorable ce temps de rédaction inattendu. Or, le déséquilibre susmentionné entre la sphère personnelle et la sphère académique peut aussi entraîner, selon notre propre expérience, un accroissement significatif des heures accordées au travail. Pour certain'e·s, confiné·e•s au domicile, cet accroissement peut se révéler malsain alors que la vision temporelle liée au travail n'est plus la même qu'à l'habitude. Considérant que les étudiant·e's n'ont plus à se déplacer pour se rendre à leurs bureaux, à la bibliothèque ou dans 
un café pour y travailler, ils n'ont plus de points de repère pour prendre des moments d'arrêt. Pour ainsi dire, le moment d'arrêt n'est plus régi par les déplacements obligatoires ou même, la fermeture de l'établissement d'enseignement, mais bien par la personne elle-même, ce qui n'est pas une tâche simple.

De surcroit, puisque la plupart des activités de lecture et de rédaction menées aux cycles supérieurs peuvent être réalisées à domicile, plusieurs répercussions ne tardent pas à se faire ressentir chez ces étudiant•e's dont le temps consacré au travail est à redéfinir: fatigue accumulée, alimentation insuffisante ou négligée, heures de sommeil revues à la baisse, sédentarité accrue, exposition prolongée aux écrans, etc. En bref, plusieurs étudiant·e's aux cycles supérieurs en sciences de l'éducation ressentent une pression immense à performer et se retrouvent, nous y compris à certains moments, à avoir de la difficulté à s'arrêter ou à ressentir un sentiment de culpabilité lors des moments d'arrêt. Cela s'ajoute à l'instantanéité technologique mise en relief par la multiplication des outils utilisés (courriel, téléphone, plateforme Web de communication, etc.) et le sentiment de toujours devoir être disponible, ce qui au bout du compte peut accentuer davantage ce déséquilibre. Pour des étudiant·e·s désirant faire carrière à l'université, l'impression de devoir toujours être présent·e·s, disponibles et prêt·e·s à travailler n'a jamais semblé plus grande.

\section{Les conditions de travail : ressources universitaires et technologiques}

À la lumière de notre expérience, nous estimons qu'un second axe phare, en l'occurrence les conditions de travail, mérite d'être aussi abordé afin de brosser un portrait des influences et des répercussions de cette pandémie sur les apprentissages et sur le parcours universitaire des étudiant·e•s. D'entrée de jeu, les conditions de travail aux cycles supérieurs nécessitent une grande capacité d'adaptation de la part des étudiant'e's, ce qui est d'autant plus vrai en situation pandémique. Ainsi, une question s'impose d'elle-même: de quelle façon cette situation affecte-t-elle concrètement les conditions de travail des étudiant·e's ? Deux éléments de réponse sont proposés en lien avec l'accessibilité aux ressources universitaires sur le plan physique ainsi que les ressources numériques.

\section{L'impossibilité d'accéder aux ressources universitaires sur le plan physique}

D'entrée de jeu, la fermeture soudaine des universités en présentiel due à la COVID-19 entraîne plusieurs changements relatifs à l'accessibilité des ressources universitaires sur le plan physique. De fait, pour la plupart des étudiant'e•s aux cycles supérieurs en sciences de l'éducation, les laboratoires et les bureaux de recherche partagés à l'université représentent les principaux espaces de travail utilisés au quotidien. Ces espaces à la fois personnels et communs sont jugés optimaux pour les étudiant ${ }^{\cdot} \cdot s-c^{-}$chercheur $^{*} \cdot s$, entre autres afin qu'ils en apprennent davantage sur les rouages de la recherche scientifique, tout en favorisant également la socialisation et la collaboration par des échanges, des discussions et du soutien entre collègues et avec la direction de recherche (Couillard, Auclair, Duchaine, 2018 ; Gingras et Gemme, 2006). Cela dit, ces espaces de travail étant inaccessibles dans ce contexte de pandémie, tout comme plusieurs points de service jugés essentiels aux études (p. ex., les bibliothèques, les locaux de réunions, le centre multimédia, les locaux associatifs, etc.), cela influence de façon inévitable les étudiant·e·s qui se retrouvent alors à court d'options. La plupart des étudiant·e's doivent sont contraints à travailler de la maison, et par le fait même, à repenser leur environnement. Dans plusieurs cas, la situation ne

4 - Formation et profession 28(4 hors-série), 2020 
fournit pas des conditions de travail jugées optimales en raison notamment des distractions, comme nous l'avons mentionné précédemment. L'horaire de travail, correspondant autrefois à une case plus rigide, doit alors se mêler à la réalité vécue par chaque étudiant·e et aux particularités de leur situation personnelle et familiale.

En plus de cette impossibilité à accéder à ces espaces de travail, les étudiant·e•s ont difficilement accès aux ressources matérielles qui s'y trouvent. Ces ressources sont pour le moins essentielles au maintien et à la poursuite de leurs activités académiques et de recherche. À titre d'exemple, il peut s'agir de logiciels de codage, de ressources audiovisuelles ainsi que de livres inédits. La plupart de ces ressources nécessitent un investissement financier de plusieurs centaines de dollars, un investissement que bon nombre d'étudiant' $e \cdot s$ aux cycles supérieurs ne peuvent se permettre, ce qui explique qu'ils les utilisent par le biais de leur établissement d'enseignement en temps normal. Ainsi, l'accessibilité limitée aux espaces de travail universitaire et aux ressources matérielles qui s'y trouvent est susceptible d'influencer les apprentissages et le parcours universitaire des étudiant'e's aux cycles supérieurs en sciences de l'éducation en contexte de pandémie.

\section{Les inégalités quant à la possession et aux usages du numérique}

Outre l'environnement physique mentionné précédemment, nous observons d'autres défis liés aux conditions de travail des étudiant'e's aux cycles supérieurs en sciences de l'éducation, entre autres relatifs aux technologies. De fait, cette pandémie entraîne un virage sans précédent vers le numérique alors que la plupart des universités misent sur l'enseignement à distance et l'apprentissage en ligne. Toutefois, notre proximité avec la communauté étudiante nous permet d'observer une exacerbation de la fracture numérique déjà bien présente dans les milieux universitaires avant la crise. Il existe selon nous un fossé grandissant quant aux inégalités relatives à la possession d'outils numériques et à l'utilisation des technologies de l'information et de la communication (TIC). Plus que jamais, posséder les outils numériques adéquats s'avère essentiel afin de collaborer, de communiquer, de produire du contenu, afin de résoudre des problèmes, d'innover et de créer (Ministère de l'Éducation et de l'Enseignement supérieur, 2019). À la lumière de cette réorganisation des conditions de travail, les étudiant'e•s qui ne possèdent pas les outils technologiques nécessaires voient leur évolution académique limitée, voire compromise. Dans certains milieux universitaires, cet enjeu est également connu et des mesures exceptionnelles sont mises en place pour répondre aux besoins des étudiant·e·s. À titre d'exemple, l'Université de Montréal (2020) a procédé à l'achat de plus de 200 ordinateurs portables et autres outils technologiques destinés à la communauté étudiante afin de contrer les effets négatifs liés à la COVID19. Cela dit, puisque l'offre demeure inférieure à la demande, des influences et des répercussions relatives à cet élément d'intérêt sont à envisager à court et à long terme.

Toujours dans cette optique, l'exacerbation de la fracture numérique que nous observons concerne aussi les différents usages technologiques : maîtrise des fonctionnalités des outils, des logiciels, des applications et des plateformes (Karsenti, 2019). La compétence numérique et les habiletés technologiques jouent un rôle déterminant dans les apprentissages et le parcours universitaire des étudiant'e's aux cycles supérieurs en sciences de l'éducation lors de la pandémie alors que leurs capacités sont sollicitées pour mener à bien leurs différentes activités. Pour certains étudiant·e's qui possèdent d'ores et déjà bon nombre de compétences et d'habiletés technologiques ou du moins, l'autonomie nécessaire pour les 
développer, le contexte exceptionnel vécu est susceptible de contribuer à la bonification de celles-ci. Cependant, le fossé entre les étudiant'e's maîtrisant ces compétences numériques et ceux éprouvant encore des difficultés est grandement exacerbé. Pour ces étudiant·e•s ne disposant pas des compétences et des habiletés technologiques nécessaires, le contexte pandémique est susceptible d'entraîner du stress, de la frustration ainsi qu'un sentiment d'infériorité et d'incapacité, et ce, dans un milieu déjà compétitif et concurrentiel.

\section{Les tâches académiques principales et secondaires}

En plus des influences et des répercussions susmentionnées sur la santé psychologique et les conditions de travail, cette pandémie mondiale entraîne, selon nous, une redéfinition des principales tâches académiques chez les étudiant'e's universitaires aux cycles supérieurs en sciences de l'éducation. A priori, le parcours universitaire, plus particulièrement dans le domaine de la recherche, est centré sur la rédaction d'un mémoire ou d'une thèse. En poursuivant une scolarité à temps plein, deux ans sont nécessaires en moyenne à la maîtrise et quatre ans au doctorat pour mener à terme ses études. Cela dit, en temps normal, force est de constater que bon nombre d'étudiant·e's en sciences de l'éducation doivent prolonger de quelques mois, voire de quelques années, leur scolarité pour y parvenir. Dans cette optique, l'enquête menée par le ministère de l'Enseignement supérieur, de la Recherche et de la Science en collaboration avec le ministère de l'Éducation, du Loisir et du Sport (2015) fait état que la principale raison d'un prolongement de scolarité est la charge de travail trop grande. Lors de la pandémie de la COVID-19, nous sommes d'avis que ladite charge de travail trop grande est susceptible d'être remise en perspective et reconsidérée par quelques étudiant···s.

\section{Le report ou l'annulation de tâches secondaires}

D'entrée de jeu, précisons que les tâches et les responsabilités des étudiant·e·s aux cycles supérieurs en sciences de l'éducation se limitent rarement à la rédaction d'un mémoire ou d'une thèse. Au fil $\mathrm{du}$ parcours universitaire, bien d'autres tâches connexes et tout aussi déterminantes viennent se greffer à la tâche principale, entre autres des activités de rayonnement, d'enseignement, de recherche

et de bénévolat. À titre d'exemple, on dénombre les charges de cours, l'assistanat au sein de projets de recherche, l'implication étudiante auprès d'associations étudiantes, la rédaction et la publication d'articles, la participation à des colloques ainsi qu'à des congrès et bien plus. Cela étant dit, le contexte pandémique exceptionnel vécu par les étudiant·e·s lors du trimestre d'hiver et d'été est susceptible de remettre en perspective la place accordée à la tâche principale et aux tâches secondaires.

De fait, en sciences de l'éducation, la majorité des recherches ont été interrompues considérant la fermeture des établissements d'enseignement primaire, secondaire, collégial et universitaire. Ainsi, n'ayant plus accès à ces terrains de recherche, bon nombre d'étudiants n'ont eu d'autre choix que de prendre une pause obligée de collecte de données ou d'autres travaux de recherche. En outre, bien que quelques colloques et congrès aient été transposés en ligne, la plupart de ces évènements scientifiques ont plutôt été annulés, libérant ainsi du temps de préparation associé à une présentation scientifique et à la participation à ces évènements. Plusieurs évènements et rencontres associatifs ont également été annulés, libérant encore une fois du temps dans les horaires des étudiant·e·s aux cycles supérieurs. De cette façon, le report ou l'annulation de plusieurs tâches ou activités de rayonnement, d'enseignement, de

6 - Formation et profession 28(4 hors-série), 2020 
recherche et de bénévolat entraînent chez bon nombre d'étudiants-chercheur·e's aux cycles supérieurs une réorganisation des tâches et leur permettent de se concentrer davantage sur la rédaction de leur mémoire ou de leur thèse.

\section{Synthèse et conclusion}

Dans cet article, nous avons tenté de mettre en lumière la situation des étudiant'• $\cdot s$ aux cycles supérieurs en sciences de l'éducation à lère de la pandémie mondiale liée à la COVID-19. Nous avons divisé les principales préoccupations en trois axes principaux : les effets psychologiques, les conditions de travail ainsi que l'impact sur les diverses tâches afin de brosser un portrait représentatif des derniers mois (figure 1).

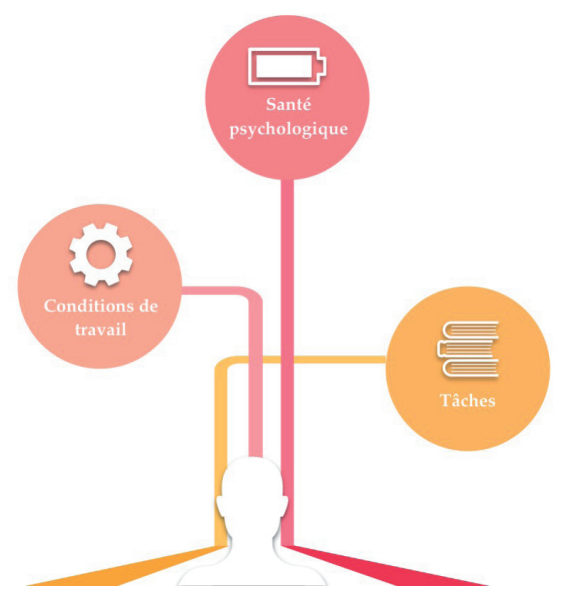

\section{Figure 1}

Les axes touchés par la pandémie de COVID-19

Pour certains, ce «trop-plein de temps » semble bénéfique pour leurs travaux de rédaction : nous pensons en effet que plusieurs mémoires et thèses se finaliseront au cours de l'automne 2020, en partie grâce à cette situation exceptionnelle. Cependant, pour d'autres, cette période est avant tout angoissante, entraînant parfois un ralentissement de la productivité. De plus, faisant partie de l'équipe de l'ACSE et des membres-étudiant'e's du Centre de recherche interuniversitaire sur la formation et la profession enseignante (CRIFPE), nous savons à quel point le réseautage et le soutien social sont importants lors du parcours universitaire. Le contact récurrent avec les autres étudiant·e•s, les professionnels de recherche, les bibliothécaires ou encore les professeur·e·s de la Faculté fait partie intégrante de notre cheminement et au bout du compte, c'est probablement ce qui s'est avéré le plus difficile lors de cette période pandémique.

Il est impossible pour le moment de connaître l'évolution de la pandémie et ses effets à long terme sur le système éducatif québécois et l'impact que cela aura sur les apprentissages et le cheminement académique des étudiant'e's aux cycles supérieurs en sciences de l'éducation. Toutefois, il est évident que cette situation nous amène à réfléchir et à redéfinir notre rôle et nos responsabilités en tant 
qu'étudiant·e•s. De nombreux changements devront être apportés, mais contribueront certainement au développement de plusieurs secteurs comme le soutien et le bien-être psychologique des étudiant·e·s ainsi que l'intégration technologique à plusieurs approches pédagogiques.

\section{Note}

1 Pour lire tous les billets, nous vous invitons sur le site du Centre d'études sur le stress humain (CESH) (https://www.stresshumain.ca/notre-impact/).

\section{Références}

Couillard, A., Auclair, R et Duchaine, S. (2018). Enquête réseau sur l'encadrement aux cycles supérieurs. Université du Québec. http://www.uquebec.ca/dri/publications/rapports_de_recherche/rapport_uq_enquete_encadrement_final_2018.pdf

Gingras, Y. et Gemme, B. (2006). L'emprise du champ scientifique sur le champ universitaire et ses effets. Actes de la recherche en sciences sociales, (4), 51-60.

Gouvernement du Québec. (2019). Rapport annuel 2018-2019._https://cdn-contenu.quebec.ca/cdn-contenu/adm/min/ education/publications-adm/rapport-annuel-de-gestion/MEES_RAG_2018-2019.PDF?1570037423

Holman, E. A., Garfin, D. R., et Silver, R. C. (2014). Media's role in broadcasting acute stress following the Boston Marathon bombings. Proceedings of the National Academy of Sciences, 111(1), 93-98.

Johnston, W. M., et Davey, G. C. (1997). The psychological impact of negative TV news bulletins: The catastrophizing of personal worries. British Journal of Psychology, 88(1), 85-91.

Karsenti, T. (2019). Le numérique en éducation : pour développer des compétences. Presses de l'Université du Québec.

Lupien, S. (2020). Par amour du stress (2 éd.). Éditions Va Savoir.

Lin, EJ., Sun, M., Choi, EY., Magee, D., Stets, CW., During, MJ. (2015). Social overcrowding as a chronic stress model that increases adiposity in mice. Psychoneuroendocrinology, 51, 318-330. DOI : 10.1016/j.psyneuen.2014.10.007

Ministère de l'Enseignement supérieur, de la Recherche et de la Science et ministère de l'Éducation, du Loisir et du sport. (2015). Enquête sur les conditions de vie des étudiantes et des étudiants. http://www.afe.gouv.qc.ca/fileadmin/AFE/documents/Publications/AFE/PUBL_enquete_conditions_vie_2013.pdf

Ministère de l'Éducation et de l'Enseignement supérieur. (2019). Cadre de référence de la compétence numérique. Repéré à : http://www.education.gouv.qc.ca/fileadmin/site_web/documents/ministere/Cadre-reference-competence-num.pdf

UNESCO. (juin 2020). Éducation : de la fermeture des établissements scolaire à la reprise. https://fr.unesco.org/covid19/ educationresponse

Université de Montréal. (juin, 2020). COVID-19 : l'UdeM met plus d'un million de dollars à la disposition de la communauté étudiante. UdeMnouvelles.

https://nouvelles.umontreal.ca/article/2020/04/07/1-udem-met-plus-d-1m-a-la-disposition-de-la-communaute-etudiante/

\section{Pour citer cet article}

Cuerrier, M. Aubin, A.-S. et Tremblay-Gagnon, D. (2020). Influences et répercussions de la pandémie sur les apprentissages et le parcours universitaire des étudiant·e's aux cycles supérieurs en sciences de l'éducation : retour d'expérience de trois doctorantes. Formation et profession, 28 (4 hors-série), 1-8. http://dx.doi.org/10.18162/fp.2020.698 